\title{
TIDAL SPLITTING OF COMETS IN EARTH'S VICINITY
}

\author{
H. RICKMAN \\ Astronomiska observatoriet \\ Box 515, S-75120 Uppsala, Sweden \\ AND \\ J.M. GREENBERG \\ Laboratory Astrophysics \\ University of Leiden, Postbus 9504, 2300 RA Leiden, The Netherlands
}

\begin{abstract}
We present a study of the influence of tidal splitting In order to clarify the role of comets as Earth impactors and bringers of organics to the young planet. Based on low values for the density and material strength, as indicated by Comet Shoemaker-Levy 9 , we have modeled the evolution of a comet in an orbit leading to Earth impact. We find that cometary nuclei impacting the Earth with radii $\gtrsim 0.1-0.3 \mathrm{~km}$ will split just before doing so. Nuclei of several $\mathrm{km}$ radius will split when passing within a distance $\lesssim 5-8$ Earth radii. Cometary nuclei with radii $\gtrsim 1 \mathrm{~km}$ stand a much larger chance of being tidally split than of hitting the Earth. Consequently, streams of fragments and debris form in Earth-crossing orbits more often than large nuclei cause impacts. The mass distribution of impacting material is shifted toward sub-km objects, whereby the survivability of organics may be significantly increased. If the cometary impactors move in orbits where $\gtrsim 5$ passages within Earth's Roche zone are expected, we estimate that most of the material reaching Earth's surface will appear in the form of split fragments.
\end{abstract}

\section{Introduction}

The total mass of Earth impactors during the first $\sim 700 \mathrm{Ma}$ after the Moon-forming event has been estimated (Delsemme 1996; Chyba and Sagan 1996) as $\sim 10^{22} \mathrm{~kg}$. The extended timescale of this bombardment points to comets coming from the outer regions of the planetary system. Comets contain a significant fraction of organic refractories in the shape of "CHON" material, consistent with Greenberg's model of the interstellar grains of star forming regions (Greenberg and Hage 1990). Mass spectroscopy of CHON material has revealed the presence of complex molecules of possibly prebiotic nature (Kissel et al. 1996).

Such crganics may have contributed very importantly to the total inventory of prebiotic material, but a critical issue is the size-dependent survivability of cometary organics upon impact. For impactors from $100 \mathrm{~m}$ to $10 \mathrm{~km}$ diameter the fraction of surviving organics decreases with increasing size, and with this in mind we will now take a first look at the effects of tidal splitting on the size distribution of cometary impactors.

\section{Critical Splitting Distance}

Comet Shoemaker-Levy 9 (to be referred to as 'SL-9') split into a dozen major fragments as it passed through Jupiter's Roche sphere in July 1992. Further fragmentation later on (Sekanina et al. 1997) caused the number of observed subnuclei to reach more than 20. The material strength $S$ can be estimated from:

$$
\frac{\Delta_{S J}}{R_{J}}=C\left(\frac{\rho}{\rho_{J}}\right)^{-1 / 3}\left(1+\frac{S}{P_{c}}\right)^{-1 / 3}
$$




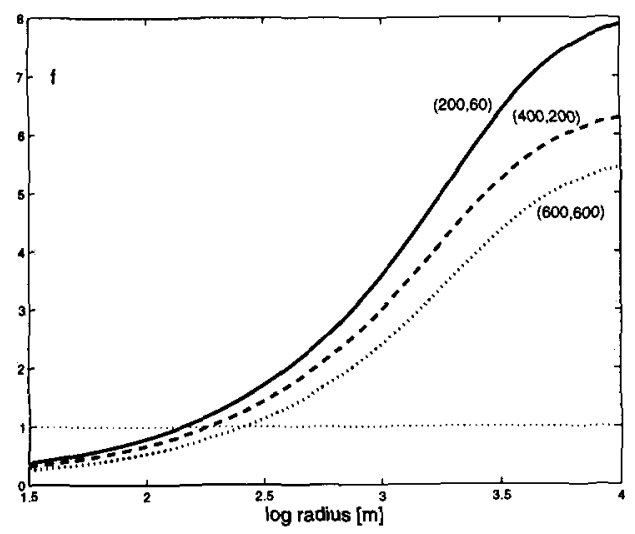

Figure 1. Splitting distance, in Earth radii, as a function of the radius of a cometary nucleus for three different combinations of (density, material strength) as indicated for each curve.

where $P_{c}=\frac{2}{3} \pi G \rho^{2} R^{2}$ is the central gravitational pressure. $\Delta_{S J} / R_{J}$ is the splitting distance expressed in jovian radii, and $\rho$ and $R$ are the cometary density and radius, respectively. For splitting near perijove $\left(\Delta_{S J} / R_{J} \simeq 1.35\right.$ ), and estimating $C=2.7$ (see Harris 1996) and $R \sim 1 \mathrm{~km}$ for the major fragments, one finds: $S \sim 100 \mathrm{~Pa}$ for $\rho \sim 300 \mathrm{~kg} / \mathrm{m}^{3}$ as estimated for $1 \mathrm{P} /$ Halley by Rickman (1989).

Greenberg et al. (1995) estimated the strength of a porous aggregate of submicron-sized, icemantled grains typical of the expected constituents of cometary material. The van der Waals bonding energy per unit volume was thus computed from the number of $\mathrm{H}_{2} \mathrm{O}$ molecules in the regions of contact between neighbouring grains. The resulting relation between porosity and strength is consistent with the above values for $\rho$ and $S$.

Eq. (1) may be used with the sole change from Jupiter's radius and density to those of the Earth in order to compute the splitting distance $f=\Delta_{S E} / R_{E}$ as a function of $R$ for different values of $\rho$ and $S$. Any comet with the assumed characteristics might split if its perigee distance were less than $\Delta_{S E}$, based on the experience from SL-9. Some results are shown in Fig. 1. At large radii, the curves level off toward limiting values that represent the Roche limits for the respective densities. The plot also shows that $f=1$ (splitting limit at Earth's surface) occurs for $R$ between 140 and $260 \mathrm{~m}$, based on the considered ranges of $\rho$ and $S$. Some extra uncertainty comes from an unavoidable error bar on $C$ due to the uncertainties of the splitting mechanism (see Sect. 3).

This indicates that cometary impactors with radii $\gtrsim 1 \mathrm{~km}$ would split during the inbound passage of the Roche sphere. Taken at face value, these results even imply that every $\mathrm{km}$-sized cometary impactor breaks up into pieces of $\$ 0.5 \mathrm{~km}$ diameter before reaching the ground. As a consequence, comets would not cause any cratering on our planet!

\section{Splitting Scenario}

However, the outcome of course depends on the splitting scenario, which is as yet controversial. Sekanina et al. (1994) claim that effective breakup of SL-9 did not occur until $3^{\mathrm{h}}$ after perijove. Such a delay would not leave time enough for breakup before impact on Earth (time of flight through Earth's Roche sphere $\lesssim 1^{\mathrm{h}}$ ). More generally, some delay should always be expected between tidal stress and material yielding in models assuming a finite material strength, and it is indeed questionable if there would be time enough for full development of hierarchical splitting!

According to the strengthless rubble pile model of other authors (Asphaug and Benz 1996; Solem 1995), the spin state of the progenitor nucleus may have a decisive influence on the outcome. Some nuclei might hardly break up at all even if they were completely strengthless, while others might be shattered even more than SL-9. Bottke et al. (1997) have modelled the encounters of rubble-pile Earth-crossing asteroids with the Earth and found that for a multi-component splitting such as that of SL-9 to occur, a prograde spin and adequate spin axis orientation are required. Moreover, 


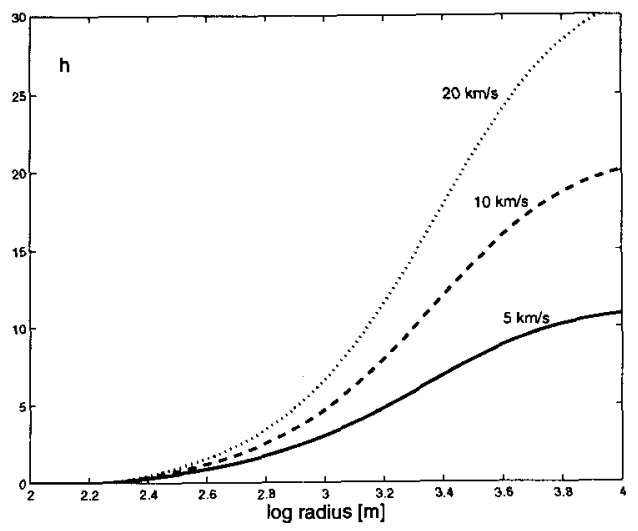

Figure 2. Ratio of cross-sections for splitting vs impact as a function of nuclear radius, assuming the intermediate values of density and strength of Fig. 1, for three different encounter velocities with respect to the Earth.

very slow encounters or very close approaches are anyway needed. Thus, in their scenario tidal splittings within Earth's Roche sphere are less important than we find under the assumption of instant splitting.

\section{Splitting vs Impact Frequencies}

In the range of radii where $f>1$, comets may pass close enough to Earth to be tidally split, without actually hitting it. Such encounters would be like the one of SL-9 with Jupiter in 1992 , and breakup would be more likely to ensue even if delayed. Fig. 1 showed that, for large radii, the splitting cross-section is much larger than the collision cross-section.

To evaluate the frequency of splittings relative to impacts, we have to account for gravitational focussing. In terms of the above-defined value of $f$, the ratio of cross-sections for splitting and impact is given by:

$$
h=\left\{\begin{array}{cc}
(f-1)\left\{1+f /\left(1+2 G M_{E} / R_{E} v_{\infty}^{2}\right)\right\} & ; f \geq 1 \\
0 & ; f \leq 1
\end{array}\right.
$$

Fig. 2 shows this ratio as a function of $R$ for encounter velocities $\left(v_{\infty}\right)$ of 5,10 and $20 \mathrm{~km} / \mathrm{s}$, spanning the range from low-eccentricity Near-Earth Object (NEO) orbits to high-eccentricity cometary orbits. It is evident that cometary nuclei with radii $\gtrsim 1 \mathrm{~km}$ stand a much larger chance of being tidally split than of hitting the Earth. Judging from the SL-9 experience, streams of fragments and debris may then form in Earth-crossing orbits. This means, as time goes by, the deposition of cometary material by bodies of various sizes, typically much smaller than the progenitor nuclcus.

\section{Cometary Earth Impactors}

How much of the deposited material will result from previous tidal splittings? This depends on (a) the a priori probability for a splitting event to occur, and (b) the fraction of the resulting fragments that later collide with the Earth.

Full computations remain to be done. We present only a first approach based on Poisson statistics for the number of passages through Earth's Roche sphere, given a certain expectance $E$. For cometary NEOs with a dynamical lifetime of $\sim 10^{7}$ orbits and inclinations $\sim 10^{\circ}$, the expected number of passages is $E \sim 5$. Given an object that impacts the Earth, Fig. 3 shows the probability $p$ that it has previously undergone tidal splitting as a function of $R$ for three values of $E$. If the efficiency of fragment delivery to Earth is high, we find that most of the impactor mass originating in km-sized nuclei reaches the Earth in smaller units.

For active comets in their typical, unstable orbits, the situation is different. With a lifetime of $\sim 10^{3}$ orbits, the chance to have first a splitting, then an impact at a later encounter is practically 